DOI: $10.17516 / 1997-1397-2021-14-5-554-565$

УДК 517.9

\title{
Energy Method for the Elliptic Boundary Value Problems with Asymmetric Operators in a Spherical Layer
}

\author{
Valery V. Denisenko* \\ Semen A. Nesterov ${ }^{\dagger}$ \\ Institute of Computational Modelling SB RAS \\ Krasnoyarsk, Russian Federation
}

Received 10.01.2021, received in revised form 13.03.2021, accepted 02.04.2021

\begin{abstract}
Three-dimensional elliptic boundary value problems arising in the mathematical modeling of quasi-stationary electric fields and currents in conductors with gyrotropic conductivity tensor in domains homeomorphic to the spherical layer are considered. The same problems are mathematical models of thermal conductivity or diffusion in moving or gyrotropic media. The operators of the problems in the traditional formulation are non-symmetric. New statements of the problems with symmetric positive definite operators are proposed. For the four boundary value problems the quadratic energy functionals, to the minimization of which the solutions of these problems are reduced, are constructed. Estimates of the obtained quadratic forms are made in comparison with the form appearing in the Dirichlet principle for the Poisson equation.
\end{abstract}

Keywords: mathematical modeling, energy method, elliptic equation, asymmetric operator.

Citation: V.V.Denisenko, S.A. Nesterov, Energy Method for the Elliptic Boundary Value Problems with Asymmetric Operators in a Spherical Layer, J. Sib. Fed. Univ. Math. Phys., 2021, 14(5), 554-565. DOI: 10.17516/1997-1397-2021-14-5-554-565.

\section{Introduction}

The tree-dimensional elliptic boundary value problems with asymmetric operators arise, for example, in mathematical modeling of quasi-stationary electric fields and currents in the global conductor, consisting of the Earth's ionosphere and atmosphere, using the domain decomposition method [1]. In such models as [2], a significant simplification of the description of the D-layer of the Earth's ionosphere, lying at heights of $50-90 \mathrm{~km}$, is used. It is in this layer that the two-dimensional model, which is adequate for higher layers, is inapplicable, and the conductivity ceases to be a scalar, as in the underlying atmosphere. Therefore, in this layer, bounded by two surfaces close to concentric spheres, it is necessary to solve a three-dimensional problem of current continuity with a gyrotropic conductivity tensor. Within the framework of the decomposition method [1], the boundary conditions arise at the selected boundaries of subdomains. In the case of interest to us, they are mixed: the condition of ideal conductivity at the upper boundary of the Dlayer and the condition on the ideal insulator on the bottom. Both conditions are heterogeneous. This boundary value problem is studied in detail in this work. For three problems which differ from the main problem by the boundary conditions, the necessary changes in the formulations

\footnotetext{
*denisen@icm.krasn.ru https://orcid.org/0000-0002-3024-3746

${ }^{\dagger}$ twist3r0k@yandex.ru https://orcid.org/0000-0002-9409-5826

(c) Siberian Federal University. All rights reserved
} 
and proofs are described. We use the approach previously used for the problem in a simply connected domain with a given normal component of the current density on the boundary [3].

Note that mathematical models of thermal conductivity or diffusion in moving media can be reduced to the same boundary value problems $[4,5]$, and the equations of heat conduction or diffusion in stationary gyrotropic media differ from the electric current continuity problem only in notation.

In the present work, the quadratic energy functionals are constructed, which makes it possible to reduce the solution of the boundary value problems for a three-dimensional elliptic equation with asymmetric coefficients to minimisation of functionals. It is shown that the energy norm is equivalent to the norm of the space $W_{2}^{(1)}(\Omega)$. The corresponding estimates are obtained with specific values of the constants that will allow us to estimate the condition number of the matrix of a system of linear algebraic equations, which will arise in the numerical solution of the problem.

\section{The electric current continuity problem}

In the three-dimensional domain $\Omega$ occupied by a conductor, the electric field strength $\mathbf{E}$ and the current density $\mathbf{J}$ in the quasistationary approximation satisfy Faraday's law, charge conservation law and Ohm's law:

$$
\operatorname{rot} \mathbf{E}=\mathbf{G}, \quad \operatorname{div} \mathbf{J}=Q, \quad \mathbf{J}=\hat{\sigma} \mathbf{E},
$$

where $\mathbf{G} \neq 0$, if there is a given magnetic field that varies with time, $Q \neq 0$, if there are external currents, $\hat{\sigma}$ is the conductivity tensor. We assume that the norms of the given functions, $Q$ and the Cartesian components of $\mathbf{G}$, are bounded in the space $L_{2}(\Omega)$. All vectors in this paper are considered as column vectors, which are transformed into row vectors by transposition, denoted by the symbol $*$. The system (1) with proper boundary conditions is referred to as the electric current continuity problem [6].

The conductivity of some substances in a magnetic field, for example, plasma in the Earth's ionosphere, is a gyrotropic tensor. In Cartesian coordinates $x, y, z$ with the $z$ axis directed along the magnetic induction vector, the conductivity tensor has the form:

$$
\hat{\sigma}=\left(\begin{array}{ccc}
\sigma_{P} & -\sigma_{H} & 0 \\
\sigma_{H} & \sigma_{P} & 0 \\
0 & 0 & \sigma_{\|}
\end{array}\right) .
$$

Its components are called field-aligned $\left(\sigma_{\|}\right)$, Pedersen $\left(\sigma_{P}\right)$ and Hall $\left(\sigma_{H}\right)$ conductivities [6]. We also use the Cowling conductivity

$$
\sigma_{C}=\left(\sigma_{P}^{2}+\sigma_{H}^{2}\right) / \sigma_{P} .
$$

In the article [3] a more general form of $\hat{\sigma}$ is considered. Here we use the form (2), to obtain more accurate estimates which are important for the numerical solution of the problems. Since the passage of electric current is accompanied by dissipation of the electric energy with density $\mathbf{J}^{*} \mathbf{E}$, the symmetric part of $\hat{\sigma}$ is positive definite. For a tensor of the form (2), this means the positiveness of the diagonal elements. Excluding ideal conductors and insulators from consideration, we assume uniform in the domain $\Omega$ boundedness of all coefficients of $\hat{\sigma}$ and uniform positive definiteness. It is convenient to write these conditions in the form:

$$
\sigma_{1} \leqslant \sigma_{C} \leqslant \sigma_{2}, \quad \sigma_{1} \leqslant \sigma_{\|} \leqslant \sigma_{2},
$$


where $\sigma_{1}, \sigma_{2}$ are positive constants.

We assume that the domain $\Omega$ is bounded and homeomorphic to the spherical layer. Its outer $\Gamma$ and inner $\gamma$ boundaries are twice continuously differentiable surfaces homeomorphic to the sphere. Normal (positive outward direction) and tangent to the boundary components of vectors are marked with indices $n$ and $\tau$.

To carry out all the proofs by simple means, we assume the convexity of the surface $\Gamma$ and the boundedness of the curvature of $\gamma$. We also consider the differences of both surfaces to be limited from two spheres with a common center and not too different radii. We will give a concrete form to all these restrictions in Section 2.

As the main problem, consider a problem with boundary conditions arising in mathematical modeling of the D-layer of the ionosphere [1]. If the domain under consideration borders on an ideal insulator, then at the boundary, the normal component of $\mathbf{J}$ equals to zero. On the border with the ideal conductor the tangent components of $\mathbf{E}$ equal to zero. The conditions can be heterogeneous:

$$
\left.J_{n}\right|_{\gamma}=q,\left.\quad \mathbf{E}_{\tau}\right|_{\Gamma}=\mathbf{g},
$$

where $q, \mathrm{~g}$ are given functions. Assume that the norms of these functions are bounded in the spaces $L_{2}(\Gamma)$ and $L_{2}(\gamma)$, respectively, that is, the boundedness of the integrals of the squares of the moduli of these functions over the surfaces on which they are given.

For the solvability of the problem (1), (4) the right-hand sides must satisfy some constraints. First, it is necessary

$$
\operatorname{div} \mathbf{G}=0,
$$

since div of rot is identically equal to zero, and without (5) the first equation (1) cannot hold.

Let us calculate the components of rot normal to the boundary from the left and right sides in the second boundary condition (4):

$$
\left.\operatorname{rot}_{n} \mathbf{E}_{\tau}\right|_{\Gamma}=\left.\operatorname{rot}_{n} \mathbf{g}\right|_{\Gamma} .
$$

The resulting left side also satisfies the first equation (1). This imposes on the given functions the second condition necessary for the solvability of the problem

$$
\left.G_{n}\right|_{\Gamma}=\left.\operatorname{rot}_{n} \mathbf{g}\right|_{\Gamma} .
$$

In Section 4, a new problem will be formulated with an conjugately factorized operator by definition [7], the solution of which is the solution to the original problem (1), (4). The existence of the solution to the new problem implies the existence of a solution to the original one, but the uniqueness must be proven independently. It does not differ from the proof given in [3] for the problem in a domain homeomorphic to a ball. Heuristic considerations are also given there, allowing us to propose a new formulation and to construct a quadratic energy functional.

\section{The energy scalar product}

We consider the set of pairs of smooth functions $F, \mathbf{P}$, satisfying the conditions:

$$
\left.F\right|_{\Gamma}=0,\left.\quad P_{n}\right|_{\Gamma}=0,\left.\quad \mathbf{P}_{\tau}\right|_{\gamma}=0 .
$$

We denote with square brackets the symmetric bilinear form:

$$
\left[\left(\begin{array}{c}
u \\
\mathbf{v}
\end{array}\right),\left(\begin{array}{c}
F \\
\mathbf{P}
\end{array}\right)\right]=\int\left(\left(\begin{array}{c}
\operatorname{grad} u \\
\operatorname{rot} \mathbf{v}
\end{array}\right)^{*}\left(\begin{array}{cc}
\frac{1}{\sigma_{0}} \hat{\sigma} \hat{S} \hat{\sigma}^{*} & -\hat{\sigma} \hat{S} \\
-\hat{S} \hat{\sigma}^{*} & \sigma_{0} \hat{S}
\end{array}\right)\left(\begin{array}{c}
\operatorname{grad} F \\
\operatorname{rot} \mathbf{P}
\end{array}\right)+\operatorname{div} \mathbf{v} \operatorname{div} \mathbf{P}\right) d \Omega
$$


where $u, \mathbf{v}$ and $F, \mathbf{P}$ are the pairs of smooth functions satisfying conditions $(7), \hat{S}$ is a symmetric positive definite matrix, which we will choose later, as well as the value of the positive constant $\sigma_{0}$. This bilinear form will be used as the energy scalar product. Let us check that it has the necessary properties for this.

Consider the corresponding quadratic form. We start with the auxiliary integral

$$
\int(\operatorname{grad} F)^{*} \operatorname{rot} \mathbf{P} d \Omega .
$$

We transform the integrand:

$$
\int(\operatorname{div}(F \operatorname{rot} \mathbf{P})-F \operatorname{div} \operatorname{rot} \mathbf{P}) d \Omega
$$

The second term is identically zero. The remaining integral is transformed using the GaussOstrogradsky theorem:

$$
\oint_{\Gamma} F \operatorname{rot}_{n} \mathbf{P} d \Gamma+\oint_{\gamma} F \operatorname{rot}_{n} \mathbf{P} d \gamma .
$$

Both integrands are equal to zero due to the first and third conditions (7), respectively. Therefore, the integral (9) is equal to zero, and therefore it can be added with any coefficient to the corresponding (8) quadratic form without changing its value.

The matrix appearing in (8) is degenerate, since its upper blocks are obtained from the lower ones through multiplication by $-\hat{\sigma} / \sigma_{0}$. By adding the doubled integral (9) the matrix of the integrand quadratic form becomes equal to

$$
K=\left(\begin{array}{cc}
\frac{1}{\sigma_{0}} \hat{\sigma} \hat{S} \hat{\sigma}^{*} & -\hat{\sigma} \hat{S}+\hat{I} \\
-\hat{S} \hat{\sigma}^{*}+\hat{I} & \sigma_{0} \hat{S},
\end{array}\right),
$$

where $\hat{I}$ is the identity matrix.

To get more accurate estimates than in [3], we use a special form $\hat{\sigma}(2)$ and constraints (3), taking

$$
\sigma_{0}=\sqrt{\sigma_{1} \sigma_{2}}, \quad \hat{S}^{-1}=\left(\hat{\sigma}+\hat{\sigma}^{*}\right) / 2
$$

With this choice, $\hat{S}$ is a diagonal matrix, and the symmetric matrix $K$ in the same local Cartesian coordinates as (2) takes the form:

$$
K=\left(\begin{array}{cccccc}
\frac{\sigma_{C}}{\sigma_{0}} & 0 & 0 & 0 & \frac{\sigma_{H}}{\sigma_{P}} & 0 \\
0 & \frac{\sigma_{C}}{\sigma_{0}} & 0 & -\frac{\sigma_{H}}{\sigma_{P}} & 0 & 0 \\
0 & 0 & \frac{\sigma_{\|}}{\sigma_{0}} & 0 & 0 & 0 \\
0 & -\frac{\sigma_{H}}{\sigma_{P}} & 0 & \frac{\sigma_{0}}{\sigma_{P}} & 0 & 0 \\
\frac{\sigma_{H}}{\sigma_{P}} & 0 & 0 & 0 & \frac{\sigma_{0}}{\sigma_{P}} & 0 \\
0 & 0 & 0 & 0 & 0 & \frac{\sigma_{0}}{\sigma_{\|}}
\end{array}\right) .
$$

The eigenvalues of the matrix do not change with the simultaneous permutation of rows and corresponding columns. This allows us to reduce the matrix $K$ to a block-diagonal form with blocks

$$
\left(\begin{array}{cc}
\frac{\sigma_{C}}{\sigma_{0}} & \frac{\sigma_{H}}{\sigma_{P}} \\
\frac{\sigma_{H}}{\sigma_{P}} & \frac{\sigma_{0}}{\sigma_{P}}
\end{array}\right), \quad\left(\begin{array}{cc}
\frac{\sigma_{C}}{\sigma_{0}} & -\frac{\sigma_{H}}{\sigma_{P}} \\
-\frac{\sigma_{H}}{\sigma_{P}} & \frac{\sigma_{0}}{\sigma_{P}}
\end{array}\right), \quad \frac{\sigma_{\|}}{\sigma_{0}}, \frac{\sigma_{0}}{\sigma_{\|}} .
$$


The eigenvalues of the two matrices are equal. The bigger of them $\lambda_{\max }$ does not exceed the matrix trace that is equal to

$$
\frac{\sigma_{C}}{\sigma_{0}}+\frac{\sigma_{0}}{\sigma_{P}}
$$

Due to (3), the eigenvalues of blocks and the last two numbers (11) do not exceed

$$
\lambda_{\max } \leqslant 2 \sqrt{\sigma_{2} / \sigma_{1}} .
$$

The determinants of the blocks (11) are equal to one, which means that smaller eigenvalues

$$
\lambda_{\min }=1 / \lambda_{\max } \geqslant \sqrt{\sigma_{1} / \sigma_{2}} / 2 .
$$

Since the eigenvalues are not changed when the coordinate system is rotated, the parameter (12) for all points of the domain $\Omega$ estimates the eigenvalues of the matrix $K$ from above, and the inverse value from below. Therefore, the condition number of the matrix $K$ does not exceed $4 \sigma_{2} / \sigma_{1}$.

By adding an auxiliary integral (9), we have not changed the value of the quadratic form (8). Therefore, from (12), (13), the upper and the lower estimations follow:

$$
\begin{aligned}
& {\left[\left(\begin{array}{c}
F \\
\mathbf{P}
\end{array}\right),\left(\begin{array}{c}
F \\
\mathbf{P}
\end{array}\right)\right] \leqslant \sqrt{\frac{4 \sigma_{2}}{\sigma_{1}}} \int\left((\operatorname{grad} F)^{2}+(\operatorname{rot} \mathbf{P})^{2}+(\operatorname{div} \mathbf{P})^{2}\right) d \Omega,} \\
& {\left[\left(\begin{array}{c}
F \\
\mathbf{P}
\end{array}\right),\left(\begin{array}{c}
F \\
\mathbf{P}
\end{array}\right)\right] \geqslant \sqrt{\frac{\sigma_{1}}{4 \sigma_{2}}} \int\left((\operatorname{grad} F)^{2}+(\operatorname{rot} \mathbf{P})^{2}+(\operatorname{div} \mathbf{P})^{2}\right) d \Omega .}
\end{aligned}
$$

Now consider the functions $F$ and $\mathbf{P}$ separately in order to estimate the right-hand side (15) from below. Since the function $F$ is equal to zero on the surface $\Gamma(7)$, it satisfies the Friedrichs inequality

$$
\int F^{2} d \Omega \leqslant c_{0} \int(\operatorname{grad} F)^{2} d \Omega
$$

where the constant $c_{0}$ is determined only by the shape of the domain and does not depend on the specific function $F$. Usually this inequality is formulated for functions equal to zero on the entire boundary, however, it suffices equality to zero on a segment of finite area (the theorem on equivalent norms [8]). For the spherical layer under consideration, it is possible to obtain a specific value of the constant $c_{0}$ by the same method, which is used for the more complex case of vector functions in [9].

In [9] for the functions $\mathbf{P}$ satisfying the boundary conditions (7), the following inequalities are proved

$$
\begin{array}{r}
\int|\mathbf{P}|^{2} d \Omega \leqslant c_{1} \int|\operatorname{grad} \mathbf{P}|^{2} d \Omega, \\
c_{2} \int|\operatorname{grad} \mathbf{P}|^{2} d \Omega \leqslant \int\left((\operatorname{rot} \mathbf{P})^{2}+(\operatorname{div} \mathbf{P})^{2}\right) d \Omega,
\end{array}
$$

where $|\operatorname{grad} \mathbf{P}|^{2}$ is the sum of the squared moduli of the gradients of all Cartesian components of $\mathbf{P}$, and the constants $c_{1}, c_{2}$ are determined only by the shape of the domain $\Omega$ and do not depend on the specific function $\mathbf{P}$.

The first inequality is similar to the Friedrichs inequality for the scalar functions which are equal to zero at the boundary. If the entire vector $\mathbf{P}$ is equal to zero at the boundary, for each of its Cartesian components one can use Friedrichs inequality to obtain the required inequality. 
However, of interest are the functions with only normal or only tangent components equal to zero at the boundary. In [10], both inequalities were proved for the functions with one of these conditions posed at the entire boundary of an arbitrary multiply connected domain. For mixed boundary conditions (7) new proofs are required.

To restrict ourselves to simple means, in [9] the additional restrictions are imposed on the domain shape: convexity of the surface $\Gamma$ and bounded curvature of $\gamma$, and also the limited difference of both surfaces from two spheres with a common center and not too different radii. Let $R$ denote the minimum radius of curvature of $\gamma$. Let the surfaces $\Gamma$ and $\gamma$ be defined in spherical coordinates using the functions $R_{\Gamma}(\theta, \varphi)$ and $R_{\gamma}(\theta, \varphi)$. We assume that

$$
0<R_{1} \leqslant R_{\gamma}(\theta, \varphi) \leqslant R_{2}, \quad 0<R_{\Gamma}(\theta, \varphi)-R_{\gamma}(\theta, \varphi) \leqslant \delta R,
$$

and the constants $R, R_{1}, R_{2}, \delta R$ will be subject to one more general restriction.

Another condition limits the angle between the boundary normal and the radial direction at each point. Let us write it down in a convenient form as a constraint on the radial component of the unit vector of the outer normal:

$$
n_{\Gamma, r} \geqslant \xi_{1}, \quad-n_{\gamma, r} \geqslant \xi_{1} .
$$

We also require that the scalar product of the normals $\mathbf{n}_{\Gamma}$ and $\mathbf{n}_{\gamma}$, calculated on one ray is positive:

$$
\mathbf{n}_{\Gamma}(\theta, \varphi) \cdot \mathbf{n}_{\gamma}(\theta, \varphi) \geqslant \xi_{2}>0 .
$$

Then the constant obtained in [9],

$$
c_{2}=1-\frac{2 R_{2}^{2} \delta R}{\xi_{1} \xi_{2}^{2} R_{1}^{2} R} .
$$

The inequality (18) makes sense only for positive $c_{2}$, which gives one more general constraint on the values of geometric parameters. In mathematical modeling of the D-layer of the ionosphere, this condition is satisfied by a large margin: the fraction in (19) is less than 0.02. If the boundaries $\gamma$ and $\Gamma$ are the spheres with radii $R_{1}$ and $R_{1}+\delta R$, then $R_{2}=R=R_{1}, \xi_{1}=\xi_{2}=1$, and the only condition $2 \delta R<R_{1}$ is enough.

Denoting by $c_{4}$ the lesser of the constants $1 / c_{0}, c_{2} / c_{1}$ in the inequalities (15)-(18) we obtain an inequality that means positive definiteness of the bilinear form (8)

$$
\left[\left(\begin{array}{c}
F \\
\mathbf{P}
\end{array}\right),\left(\begin{array}{c}
F \\
\mathbf{P}
\end{array}\right)\right] \geqslant c_{4} \sqrt{\frac{4 \sigma_{2}}{\sigma_{1}}} \int\left(F^{2}+|\mathbf{P}|^{2}\right) d \Omega .
$$

The same set of inequalities gives a lower estimate for (8) in terms of the sum of the squares of the norms of $F$ and the Cartesian components of $\mathbf{P}$ as elements of the space $W_{2}^{(1)}(\Omega)$.

It is easy to prove the inequality:

$$
(\operatorname{rot} \mathbf{P})^{2}+(\operatorname{div} \mathbf{P})^{2} \leqslant 3 \sum_{i, j=1}^{3}|\operatorname{grad} \mathbf{P}|^{2} .
$$

We expand the expressions on the left, immediately replacing the products with the sums of squares, without decreasing the value of the entire expression. Bringing similar terms, we get the sum, in which the squares of all derivatives of all components of the vector $\mathbf{P}$ enter with the coefficients 2 or 3 , while on the right-hand side (20) they all appear with coefficient 3. 
With (14) and (20) the energy norm is estimated in terms of the same norms of $F, \mathbf{P}$ from above.

The upper and lower estimates mean the equivalence of the introduced energy norm to the norm of the space $W_{2}^{(1)}(\Omega)$. In particular, this allows for the numerical solution of the problem to use the same approximating functions, as for the Poisson equation.

\section{The energy functional}

In accordance with the energy method [11], we define the energy functional:

$$
W(F, \mathbf{P})=\frac{1}{2}\left[\left(\begin{array}{c}
F \\
\mathbf{P}
\end{array}\right),\left(\begin{array}{c}
F \\
\mathbf{P}
\end{array}\right)\right]-\frac{1}{\sigma_{0}} \int F Q d \Omega-\int \mathbf{P}^{*} \mathbf{G} d \Omega+\int_{\Gamma} \mathbf{P}^{*} \mathbf{g} d \Gamma+\frac{1}{\sigma_{0}} \int_{\gamma} F q d \gamma .
$$

We use the Cauchy-Bunyakovsky inequality to estimate the linear functionals:

$$
\begin{gathered}
\left|\int F Q d \Omega\right|^{2} \leqslant \int F^{2} d \Omega \int Q^{2} d \Omega, \quad\left|\int \mathbf{P}^{*} \mathbf{G} d \Omega\right|^{2} \leqslant \int|\mathbf{P}|^{2} d \Omega \int|\mathbf{G}|^{2} d \Omega, \\
\left|\int_{\gamma} F q d \gamma\right|^{2} \leqslant \int_{\gamma} F^{2} d \gamma \int_{\gamma} q^{2} d \gamma, \quad\left|\int_{\Gamma} \mathbf{P}^{*} \mathbf{g} d \Gamma\right|^{2} \leqslant \int_{\Gamma}|\mathbf{P}|^{2} d \Gamma \int_{\Gamma}|\mathbf{g}|^{2} d \Gamma .
\end{gathered}
$$

Due to the inequalities (16), (17), the right-hand sides (22) are estimated from above by the energy norm with some factor independent of $F, \mathbf{P}$. The second factors are bounded due to belonging of $Q$ and Cartesian components of $\mathbf{G}$ to the space $L_{2}(\Omega)$, specified in the formulation of equations (1). Therefore, the first two linear functionals are bounded.

The integral $|\mathbf{P}|^{2}$ over the boundary $\Gamma$ is estimated from above in terms of the integral $|\operatorname{grad} \mathbf{P}|^{2}$ over the domain $\Omega$ in [9]. It is not difficult to estimate in a similar way the integral of $F^{2}$ over the boundary $\gamma$ in terms of the integral of $|\operatorname{grad} F|^{2}$. Since the functions $F, \mathbf{P}$ from the energy space are elements of $W_{2}^{(1)}(\Omega)$, the right-hand sides of the inequalities (23) are estimated from above by the energy norm of the pair of $F, \mathbf{P}$ with some factor independent of $F, \mathbf{P}$. The second factors in (23) are bounded due to $q$ and Cartesian components of $\mathbf{g}$ belonging to the spaces $L_{2}(\Gamma)$ and $L_{2}(\gamma)$, respectively, which was as agreed in the formulation of the boundary conditions (4). This means that the last linear functionals in (21) are also bounded.

By F. Rees's theorem, any bounded linear functional can be represented in the form of a scalar product by some uniquely defined element of Hilbert space. Therefore, the energy functional (21) can be written as

$$
W(F, \mathbf{P})=\frac{1}{2}\left[\left(\begin{array}{c}
F \\
\mathbf{P}
\end{array}\right),\left(\begin{array}{l}
F \\
\mathbf{P}
\end{array}\right)\right]-\left[\left(\begin{array}{l}
a \\
\mathbf{b}
\end{array}\right),\left(\begin{array}{l}
F \\
\mathbf{P}
\end{array}\right)\right],
$$

where $a, \mathbf{b}$ is some element of the energy space.

This expression can be transformed by highlighting the square of the difference:

$$
W(F, \mathbf{P})=\frac{1}{2}\left[\left(\begin{array}{c}
F-a \\
\mathbf{P}-\mathbf{b}
\end{array}\right),\left(\begin{array}{c}
F-a \\
\mathbf{P}-\mathbf{b}
\end{array}\right)\right]-\frac{1}{2}\left[\left(\begin{array}{c}
a \\
\mathbf{b}
\end{array}\right),\left(\begin{array}{c}
a \\
\mathbf{b}
\end{array}\right)\right] .
$$

The second term does not depend on $F, \mathbf{P}$, and the first is positive definite. Therefore the minimum of $W(F, \mathbf{P})$ is attained at $F=a, \mathbf{P}=\mathbf{b}$.

Since the element $a, \mathbf{b}$ belongs to the energy space and is defined uniquely, it is proved that in the energy space there exists, and the only one, an element that supplies the energy functional with a minimum value. 


\section{The generalized solution}

Let us introduce the notation

$$
\mathbf{E}=-\frac{1}{\sigma_{0}} \hat{S} \hat{\sigma}^{*} \operatorname{grad} F+\hat{S} \operatorname{rot} \mathbf{P}, \quad \mathbf{J}=\hat{\sigma} \mathbf{E},
$$

where $F, \mathbf{P}$ are the functions which provide the minimum value to the energy functional. Let us prove that these $\mathbf{J}, \mathbf{E}$ are the solution to the original boundary value problem (1), (4).

The condition for the minimality of the energy functional, taking into account the notation (25), can be written as an identity valid for arbitrary smooth functions $u, \mathbf{v}$ satisfying the conditions (7):

$$
\begin{array}{r}
\int\left(-(\operatorname{grad} u)^{*} \mathbf{J} / \sigma_{0}+(\operatorname{rot} \mathbf{v})^{*} \mathbf{E}+\operatorname{div} \mathbf{v} \operatorname{div} \mathbf{P}-u Q / \sigma_{0}-\mathbf{v}^{*} \mathbf{G}\right) d \Omega+ \\
+\frac{1}{\sigma_{0}} \int_{\gamma} u q d \gamma+\int_{\Gamma} \mathbf{v}^{*} \mathbf{g} d \Gamma .
\end{array}
$$

If we additionally assume the smoothness of all functions, we can use integration by parts, for transformation of this identity to the form:

$$
\begin{aligned}
& \frac{1}{\sigma_{0}} \int u(\operatorname{div} \mathbf{J}-Q) d \Omega+\int \mathbf{v}^{*}(\operatorname{rot} \mathbf{E}-\mathbf{G}) d \Omega+\int \operatorname{div} \mathbf{v} \operatorname{div} \mathbf{P} d \Omega+ \\
&+\frac{1}{\sigma_{0}} \int_{\gamma} u\left(-J_{n}+q\right) d \gamma+\int_{\Gamma} \mathbf{v}^{*}\left(-\mathbf{E}_{\tau}+\mathbf{g}\right) d \Gamma=0 .
\end{aligned}
$$

Take $u=0$ and the function $\mathbf{v}$ of the form

$$
\mathbf{v}=\operatorname{grad} V,\left.\quad V\right|_{\Gamma}=0,\left.\quad V\right|_{\gamma}=0 .
$$

Then the identity (27) takes the form:

$$
\int(\operatorname{grad} V)^{*}(\operatorname{rot} \mathbf{E}-\mathbf{G}) d \Omega+\int \operatorname{div} \operatorname{grad} V \operatorname{div} \mathbf{P} d \Omega=0 .
$$

The first integral by the Gauss-Ostrogradskii theorem is

$$
\oint_{\Gamma} V(\operatorname{rot} \mathbf{E}-\mathbf{G})_{n} d \Gamma+\oint_{\gamma} V(\operatorname{rot} \mathbf{E}-\mathbf{G})_{n} d \gamma-\int V(\operatorname{div} \operatorname{rot} \mathbf{E}+\operatorname{div} \mathbf{G}) d \Omega .
$$

The integrands are equal to zero, since $V$ is equal to zero at the boundaries $\Gamma, \gamma$ due to (28), div rot is identically zero, and the function $\mathbf{G}$ satisfies the condition (5).

Therefore, from (29) we obtain the identity

$$
\int \operatorname{div} \operatorname{grad} V \operatorname{div} \mathbf{P} d \Omega=0,
$$

which is valid for any function $V$ equal to zero on the boundary.

This identity allows us to prove the equality to zero $\operatorname{div} \mathbf{P}$ in the usual way. Assuming the opposite, take a point in $\Omega$, where $\operatorname{div} \mathbf{P} \neq 0$, and its neighborhood, where $\operatorname{div} \mathbf{P}$ is sign-preserving. We construct $V$ as a solution to the Dirichlet problem for the Poisson equation with the righthand side, equal to 1 in the selected neighborhood, and zero for the rest of $\Omega$. Substituting such a function $V$, we obtain the nonzero value of the last integral, which contradicts the identity. 
Proving that

$$
\operatorname{div} \mathbf{P}=0,
$$

the third integral in (27) can be eliminated. After that, using the arbitrariness of the functions $u, \mathbf{v}$, it is easy to prove that all factors for $u, \mathbf{v}$ are equal to zero. Since $\mathbf{E}$ and $\mathbf{J}$ are used in the present section only for the abbreviated notation of the expression (25), the following equations are fulfilled:

$$
\begin{array}{r}
\operatorname{div}\left(-\frac{1}{\sigma_{0}^{2}} \hat{\sigma} \hat{S} \hat{\sigma}^{*} \operatorname{grad} F+\frac{1}{\sigma_{0}} \hat{\sigma} S \operatorname{rot} \mathbf{P}\right)=Q / \sigma_{0}, \\
\operatorname{rot}\left(-\frac{1}{\sigma_{0}} \hat{S} \hat{\sigma}^{*} \operatorname{grad} F+\hat{S} \operatorname{rot} \mathbf{P}\right)=\mathbf{G}, \\
\left.\left(-\frac{1}{\sigma_{0}^{2}} \hat{\sigma} \hat{S} \hat{\sigma}^{*} \operatorname{grad} F+\frac{1}{\sigma_{0}} \hat{\sigma} \hat{S} \operatorname{rot} \mathbf{P}\right)_{n}\right|_{\gamma}=q, \\
\left.\left(-\frac{1}{\sigma_{0}} \hat{S} \hat{\sigma}^{*} \operatorname{grad} F+\hat{S} \operatorname{rot} \mathbf{P}\right)_{\tau}\right|_{\Gamma}=\mathbf{g} .
\end{array}
$$

The converse statement is also easy to prove: the solution of the problem (30)-(33), (7) gives the minimum value in the energy space to the energy functional. Indeed, let $F, \mathbf{P}$ be a solution to this boundary value problem. Let us write down the energy functional for the sum of the functions $F+t u, \mathbf{P}+t \mathbf{v}$, where $t-$ an arbitrary number, $u, \mathbf{v}$ are smooth functions satisfying the conditions (7):

$$
\begin{array}{r}
W(F+t u, \mathbf{P}+t \mathbf{v})=W(F, \mathbf{P})+\frac{t^{2}}{2}\left[\left(\begin{array}{l}
u \\
\mathbf{v}
\end{array}\right),\left(\begin{array}{l}
u \\
\mathbf{v}
\end{array}\right)\right]+t\left(\left[\left(\begin{array}{l}
u \\
\mathbf{v}
\end{array}\right),\left(\begin{array}{l}
F \\
\mathbf{P}
\end{array}\right)\right]-\right. \\
\left.-\int\left(u Q / \sigma_{0}+\mathbf{v}^{*} \mathbf{G}\right) d \Omega+\int_{\gamma} u q / \sigma_{0} d \gamma+\int_{\Gamma} \mathbf{v}^{*} \mathbf{g} d \Gamma\right) .
\end{array}
$$

Since $F, \mathbf{P}$ is a solution to the boundary value problem, the identity (27) is satisfied for arbitrary $u, \mathbf{v}$. By integrating by parts from (27) we obtain the identity (26), which means equality to zero of the factor of $t$ in the square trinomial (34).

The coefficient of $t^{2}$ is positive, since the positive definiteness of the energy quadratic form has been proved, and we are interested in $u, \mathbf{v}$, which are not identically zero. Therefore

$$
W(F+t u, \mathbf{P}+t \mathbf{v}) \geqslant W(F, \mathbf{P}),
$$

and equality is obtained only for $t=0$, that is, the minimum of $W$ is attained at the element $F, \mathbf{P}$.

As can be seen from (27), the constructed functions $\mathbf{E}$ and $\mathbf{J}$ satisfy equations and boundary conditions of the original boundary value problem (1), (4). As already noted, the uniqueness of the solution is proved in almost the same way as in [3].

Thus, we have proposed a new formulation of the problem (30)-(33), (7), which, unlike the original problem, has a symmetric positive definite operator.

At the beginning of the section, an additional smoothness assumption was made. If this condition is not satisfied, the identity (26) cannot be transformed into (27). In this case, the pair of functions $F, \mathbf{P}$, providing the energy functional the minimum value, we consider as the generalized solution to the problem (30)-(33), (7), and the pair $\mathbf{E}, \mathbf{J}$ constructed from them by formulae (25) is the generalized solution of the original problem (1), (4). 
The existence and uniqueness of the generalized solution of the problem (30)-(33), (7) have been proved, therefore the existence of the generalized solution of the original boundary value problem (1), (4) is proved, and the principle of the minimum of the energy functional is substantiated for it.

\section{Other boundary value problems}

Consider three problems which differ from the main problem by the boundary conditions. In the first problem, we swap the parts of the boundary $\gamma$ and $\Gamma$ in the boundary conditions (4). Then, naturally, the condition of the form (6), that is necessary for the solvability of this first problem, is moved to $\gamma$.

As described in [3] and demonstrated above when constructing the generalized solution (26), the conditions for the set of pairs of smooth functions $F, \mathbf{P}$, are the conjugate ones to the boundary conditions of the original problem. Therefore, in the conditions (7) and in the energy functional, all changes are limited to the permutation of $\gamma$ and $\Gamma$. Note that the value of the constant in the inequalities (17), (18) [9] varies.

As the second boundary value problem, consider the domain $\Omega$ surrounded by an insulator. Then the first of the conditions (4) is set on both sections of the boundary $\gamma$ and $\Gamma$. From the charge conservation law, integrated over the entire domain, a necessary condition for the solvability of this problem arises:

$$
\int Q d \Omega-\int_{\gamma} q d \gamma-\int_{\Gamma} q d \Gamma=0
$$

The conditions for the pairs of functions $F, \mathbf{P}(7)$ take the form

$$
\left.\mathbf{P}_{\tau}\right|_{\Gamma}=0,\left.\quad \mathbf{P}_{\tau}\right|_{\gamma}=0, \quad \int F d \Omega=0 .
$$

The last condition is the conjugate one to the condition (35). It has to be added, since without the equality $F=0$ on the boundary that was in (7), the Friedrichs inequality cannot be used for the function $F$. The inequality (16) with a different constant is now fulfilled as Poincare's inequality. The condition in question can be replaced by fixing some other average value of the function $F$, for example, the average over $\gamma$ or $\Gamma$. Then the inequality (16) will be true as a consequence of the equivalent normalization theorem [8]. In fact, such a condition should eliminate the ambiguity of the solution corresponding to the addition of an arbitrary constant to $F$. Inequalities (17), (18) for the vector functions $\mathbf{P}$ whose tangent components are equal to zero on the whole boundary (36), were proved in [10] for bounded multiply connected domains of general form, however, without specifying the values of the constants.

The third boundary value problem describes the domain $\Omega$ surrounded by an ideal conductor. Then, on both sections of the boundary $\gamma$ and $\Gamma$, the second of the conditions (4) is set. In contrast to the second boundary value problem, the additional solvability condition does not arise. It would arise if the domain $\Omega$ is, for example, a torus, in which this problem has a nonzero solution for zero right-hand sides. For isotropic constant conductivity in an axisymmetric torus, this would be an azimuthal electric field, not changing with displacement in the direction of the axis of symmetry and decreasing in inverse proportion to the distance to the axis, and the current density proportional to it. Of course, the nonzero Joule dissipation of such a current system must be compensated by some external sources. In this example, the energy source is a 
time-varying magnetic field in the domain encompassed by this torus. For multiply connected domains of general form, the ambiguity of solutions to similar problems is analyzed in [10].

The conditions for the set of pairs of functions $F, \mathbf{P}$ in the third boundary value problem have the form

$$
\left.F\right|_{\gamma, \Gamma}=0,\left.\quad P_{n}\right|_{\gamma, \Gamma}=0 .
$$

The inequality (16) for the function $F$ remains the Friedrichs inequality, and for the vector functions $\mathbf{P}$ with zero normal component on the entire boundary the inequalities (17), (18) are also proved in [10].

Thus, for three boundary value problems, new formulations of problems are proposed, which, in contrast to the original problems, have symmetric positive definite operators, and the principles of the minimum of energy functionals are justified for them.

Taking into account the notation (25), with the tensor $\hat{S}$ chosen in accordance with (10), the quadratic form, corresponding to the energy scalar product (8) can be written in terms of the original electric current continuity problem (1), (4):

$$
\frac{1}{\sigma_{0}} \int\left(\mathbf{E}^{*} \mathbf{J}+(\operatorname{div} \mathbf{P})^{2}\right) d \Omega .
$$

The product $\mathbf{E}^{*} \mathbf{J}$ is the Joule dissipation density, that is, heat release accompanying the passage of the electric current. Since the minimization of the energy functional means satisfaction of the equality (30), the quadratic form (37) is equal to the total Joule dissipation in the domain $\Omega$, which justifies the name "energy". Thus, the introduced energy norm makes sense from the point of view of nonequilibrium thermodynamics.

This work is supported by the Krasnoyarsk Mathematical Center and financed by the Ministry of Science and Higher Education of the Russian Federation in the framework of the establishment and development of regional Centers for Mathematics Research and Education (Agreement no. 075-02-2020-1631).

\section{References}

[1] V.V.Denisenko, Statements of the boundary value problems in mathematical simulation of a quasistationary electric field in the atmosphere and ionosphere, Journal of Physics: Conference Series, 1715(2021), no. 012016, 1-10. DOI: 10.1088/1742-6596/1715/1/012016

[2] V.V.Denisenko, M.J.Rycroft, R.G.Harrison, Mathematical Simulation of the Ionospheric Electric Field as a Part of the Global Electric Circuit, Surveys in Geophysics, 40(1)(2019), $1-35$.

[3] V.V.Denisenko, The energy method for three dimensional elliptical equations with asymmetric tensor coefficients, Siberian Mathematical Journal, 38(6)(1997), 1099-1111.

[4] V.V.Denisenko, Energy method in problems of transfer in media moving in multiply connected domains, Russian Journal of Numerical Analysis and Mathematical Modelling, 15(2)(2000), 127-143.

[5] V.V.Denisenko, Symmetric operators for transfer problems in three-dimensional moving media, Siberian Journal of Industrial Mathematics, 4(2001), no. 1, 73-82. 
[6] J.K.Hargreaves, The Upper Atmosphere and Solar-terrestrial Relations, New York, Van Nostrand Reinold, 1979.

[7] A.N.Konovalov, Conjugate-factorized models in mathematical physics problems, Novosibirsk, Computing Center SB RAS, Preprint 1095, 1997 (in Russian).

[8] S.L.Sobolev, Some applications of the functional analysis in mathematical physics, Novosibirsk: publishing house of the USSR Academy of Siences, 1962 (in Russian).

[9] V.V.Denisenko, S.A.Nesterov, Inequalities for the norms of vector functions in a spherical layer, Cornell University Library, Preprint, 2020. http://arxiv.org/abs/2010.11613

[10] E.B.Bykhovsky, N.V.Smirnov, About the orthogonal decomposition of the space of the vector-functions and about the operators of vector analysis, Reports of Steklov Moscow Mathematical Institute, 59(1960), 5-36 (in Russian).

[11] S.G.Mikhlin, Variational methods in mathematical physics, Moscow, Gostekhizdat, 1957 (in Russian).

\title{
Энергетический метод для эллиптических краевых задач с несимметричными операторами в шаровом слое
}

\author{
Валерий В. Денисенко \\ Семен А. Нестеров \\ Институт вычислительного моделирования СО РАН \\ Красноярск, Российская Федерация
}

\begin{abstract}
Аннотация. Рассмотрены трехмерные эллиптические краевые задачи, возникающие при математическом моделировании квазистационарных электрических полей и токов в проводниках с гиротропным тензором проводимости в областях, гомеоморфных шаровому слою. Аналогичные задачи формулируются при моделировании теплопроводности или диффузии в движущихся или гиротропных средах. Операторы задач в традиционной формулировке являются несимметричными. Предложены новые формулировки задач с симметричными положительно определенными операторами. Для четырех краевых задач построены квадратичные функционалы энергии, к минимизации которых сведено решение этих задач. Выполнены оценки полученных квадратичных форм в сравнении с формой, фигурирующей в принципе Дирихле для уравнения Пуассона.
\end{abstract}

Ключевые слова: математическое моделирование, энергетический метод, эллиптическое уравнение, несимметричный оператор. 